\title{
Pharmacological Characteristics and Control Efficacy of a Novel SDHI Fungicide Pydiflumetofen Against Sclerotinia sclerotiorum
}

Yabing Duan, College of Plant Protection, Nanjing Agricultural University, Nanjing, 210095, China; and State \& Local Joint Engineering Research Center of Green Pesticide Invention and Application, Nanjing, 210095, China; Qian Xiu, Haoran Li, and Tao Li, College of Plant

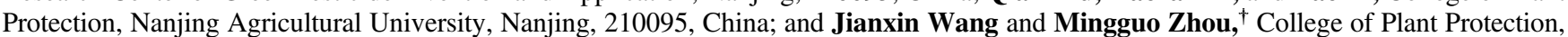
Nanjing Agricultural University, Nanjing, 210095, China; and State \& Local Joint Engineering Research Center of Green Pesticide Invention and Application, Nanjing, 210095, China

\begin{abstract}
Pydiflumetofen is a novel succinate dehydrogenase inhibitor fungicide. In the current research, we determined the sensitivity of 166 Sclerotinia sclerotiorum strains to pydiflumetofen using mycelial growth inhibition method. The results suggest that pydiflumetofen exhibited a strong inhibitory activity against $S$. sclerotiorum and the $\mathrm{EC}_{50}$ values ranged from 0.0058 to $0.0953 \mu \mathrm{g} \mathrm{ml}^{-1}$, with a mean $\mathrm{EC}_{50}$ value of $0.0250 \mu \mathrm{g} \mathrm{ml}{ }^{-1}$. However, the baseline sensitivity was not normally distributed because of a high variation factor. After treatment with pydiflumetofen, cell membrane permeability increases, but exopolysaccharide and oxalic acid production decreases, which contributes to reduced virulence of S. sclerotiorum and leads to failure of disease infection. In addition, protective and curative activity was performed on detached oilseed

rape leaves by artificial inoculation. Pydiflumetofen exhibited excellent protective and curative effects against $S$. sclerotiorum on oilseed rape, and the protective effect was better than the curative effect. Further, field trials were conducted to evaluate the potential of pydiflumetofen in controlling Sclerotinia stem rot (SSR) caused by S. sclerotiorum on oilseed rape. Compared with the currently used fungicides, pydiflumetofen not only exhibited excellent control efficacy against SSR, but also dramatically reduced the dosage of fungicides in the field. Thus, this study provides important references for revealing pharmacological mechanism of pydiflumetofen against $S$. sclerotiorum and managing SSR on oilseed rape caused by benzimidazole- and dicarboximideresistant populations.
\end{abstract}

Sclerotinia sclerotiorum, a devastating phytopathogenic fungus, can attack more than 400 cultivated plant species, such as many vegetable and economic crops, causing serious losses of yield and quality in many countries (Abawi and Grogan 1979; Bardin and Huang 2001; Boland and Hall 1994; Bolton et al. 2006; Letham et al. 1976; Purdy 1979). Due to the limit of host resistance gene resources (Bardin and Huang 2001; Boland 1997), application of fungicides is always a primary tool for controlling Sclerotinia disease on most crops (Lee et al. 2012). Unfortunately, due to the high selective pressures of extensively used fungicides, the development of fungicide resistance in S. sclerotiorum has increased in many countries, leading to control failure (Li et al. 2003; Ma et al. 2009a; Wang et al. 2014b). The benzimidazole fungicides have been extensively applied in China for controlling Sclerotinia stem rot (SSR) on oilseed rape since the early 1980s (Wang et al. 2014b). In recent years, however, the carbendazimresistant $S$. sclerotiorum populations have gradually increased under the high selective pressures of benzimidazoles, resulting in control failure (Duan et al. 2015, 2016; Ma et al. 2009a; Wang et al. 2014b). Moreover, dicarboximide fungicides have also been applied for controlling SSR in China in the past few decades. However, dicarboximide resistance has emerged in some provinces of China in recent years (Ma et al. 2009a; Wang et al. 2014b). Therefore, it is very necessary to find more effective compounds to replace the fungicides that are currently applied for controlling SSR.

Pydiflumetofen (development code number: SYN545974), 3-(Difluoromethyl)-N-methoxy-1-methyl-N-[(RS)-1-methyl-2-(2,4,6trichlorophenyl)ethyl]pyrazole-4-carboxamide (Fig. 1) (https://apvma. gov.au/sites/default/files/publication/29011-pydiflumetofen_draft_ prs-final_.pdf), is a novel fungicide developed by Syngenta (Buxton

${ }^{\dagger}$ Corresponding author: M. G. Zhou; E-mail: mgzhou@njau.edu.cn

Funding: This work was supported by the National Key Research and Development Program of China (2016YFD0200504-01), and the National Natural Science Foundation of China (31401764).

Accepted for publication 28 June 2018.

C 2019 The American Phytopathological Society et al. 2016). This fungicide belongs to a member of the succinate dehydrogenase inhibitors. To date, pydiflumetofen has been registered for use in potatoes and grapes in New Zealand, and pydiflumetofencombined products have been approved for use in soybean, peanuts, tomatoes, peppers, and grapes in Argentina (Buxton et al. 2016; Harp et al. 2016; Hou et al. 2017; Olaya et al. 2016). However, its in vitro fungicidal activity and in planta control efficacy against $S$. sclerotiorum have not been reported. Therefore, the objectives of the current research were: (i) to establish the pydiflumetofen sensitivity distributions of S. sclerotiorum populations from the different geographical regions in China; (ii) to assess the patterns of cross resistance between pydiflumetofen and other currently used fungicides; (iii) to determine the physiological characteristics of $S$. sclerotiorum as affected by pydiflumetofen; (iv) to evaluate the protective and curative effects of pydiflumetofen on oilseed rape against $S$. sclerotiorum; and (v) to test the control efficacy of pydiflumetofen in SSR on oilseed rape in the fields where carbendazim has been extensively applied for several decades.

\section{Materials and Methods}

Fungal strains and fungicides. S. sclerotiorum strains were isolated from mature sclerotia collected from the diseased oilseed rape stems from different locations in Jiangsu Province of China during 2014 and 2016. These strains were purely cultured by transferring a single mycelial tip from each colony and stored in the Fungicide Biology Laboratory, Nanjing Agriculture University.

Technical grade pydiflumetofen (99\%) was kindly provided by Syngenta (China) and dissolved in methanol as stock solution $\left(10 \mathrm{mg} \mathrm{ml}^{-1}\right)$. Pydiflumetofen formulation (200 g/liter suspension concentrate, SC) was provided by Syngenta (China). Carbendazim formulation (50\%, wettable powder, WP), dimethachlon formulation $(50 \%, \mathrm{WP})$, and iprodione formulation $(40 \%$, WP) were purchased in Nanjing, China. Technical grade fungicide was used for the tests for mycelial growth sensitivity, cell membrane permeability, exopolysaccharide and oxalic acid production, and protective and curative activity. Fungicide formulations were used for the field trials.

Fungicide sensitivity tests in vitro. The pydiflumetofen sensitivity of S. sclerotiorum was tested using mycelial growth inhibition method as described in previous studies (Duan et al. 2012, 2013a). Mycelial plugs (5 $\mathrm{mm}$ in diameter) cut from the margin of 2-day-old 
fungal colonies were placed on PDA plates containing 0, 0.001, 0.004, $0.016,0.064$, or $0.256 \mu \mathrm{g} \mathrm{ml}^{-1}$ pydiflumetofen. After incubation at $25^{\circ} \mathrm{C}$ for 2 days in the dark, colony diameters were determined and the median effective concentration $\left(\mathrm{EC}_{50}\right)$ was calculated by fitting mycelial growth inhibition rates against the log-transformed fungicide concentrations. Each concentration had four replicates for each strain.

Cross resistance. Four dimethachlon-resistant strains and four carbendazim-resistant field strains were used to determine the pattern of cross resistance as previously described (Duan et al. 2014; Wang et al. 2009, 2014a). Mycelial plugs cut from the margin of fungal colonies were placed on PDA plates amended with a series of concentrations of pydiflumetofen, dimethachlone, or carbendazim as previously described (Kuang et al. 2011). After incubation at $25^{\circ} \mathrm{C}$ for 2 days in the dark, the colony diameters were determined and the $\mathrm{EC}_{50}$ values were calculated as described above. Each concentration had four replicates for each strain.

Cell membrane permeability. Three $S$. sclerotiorum strains were used to determine cell membrane permeability at the presence of pydiflumetofen according to a previous study (Duan et al. 2013a) with minor modification. Five mycelial plugs were transferred into $100 \mathrm{ml}$ of yeast extract peptone dextrose (YEPD) media (Ge et al. 2013). After incubation for 3 days on a rotary shaker (175 rpm at $25^{\circ} \mathrm{C}$ ), fresh mycelia were harvested and washed twice with sterile deionized water. Mycelia ( $0.5 \mathrm{~g}$ ) were suspended in $20 \mathrm{ml}$ of sterile deionized water containing different concentrations of pydiflumetofen $\left(\mathrm{EC}_{50}\right.$ value and $10 \times \mathrm{EC}_{50}$ value for each strain). After incubation for $0,5,10,20,40,60,80,100,120,140,160$, or $180 \mathrm{~min}$, the electrical conductivity of each suspension was determined using the conductivity meter. After $180 \mathrm{~min}$, the suspensions were boiled for $5 \mathrm{~min}$, and final conductivity was measured. The relative conductivity of mycelia was calculated as the following formula: Relative conductivity $(\%)=$ real-time conductivity/final conductivity $\times 100$. Each treatment has four replicates for each strain and the experiment was repeated twice.

Exopolysaccharide (EPS) production. To explore the effect of pydiflumetofen on EPS production, the experiment was performed according to the phenol-sulfuric acid method (Duan et al. 2014) with minor modification. Mycelial plugs were transferred into 250-ml conical flasks containing $100 \mathrm{ml}$ YEPD media. After culturing for $36 \mathrm{~h}$ on a rotary shaker $\left(175 \mathrm{rpm}\right.$ at $\left.25^{\circ} \mathrm{C}\right)$, pydiflumetofen at the

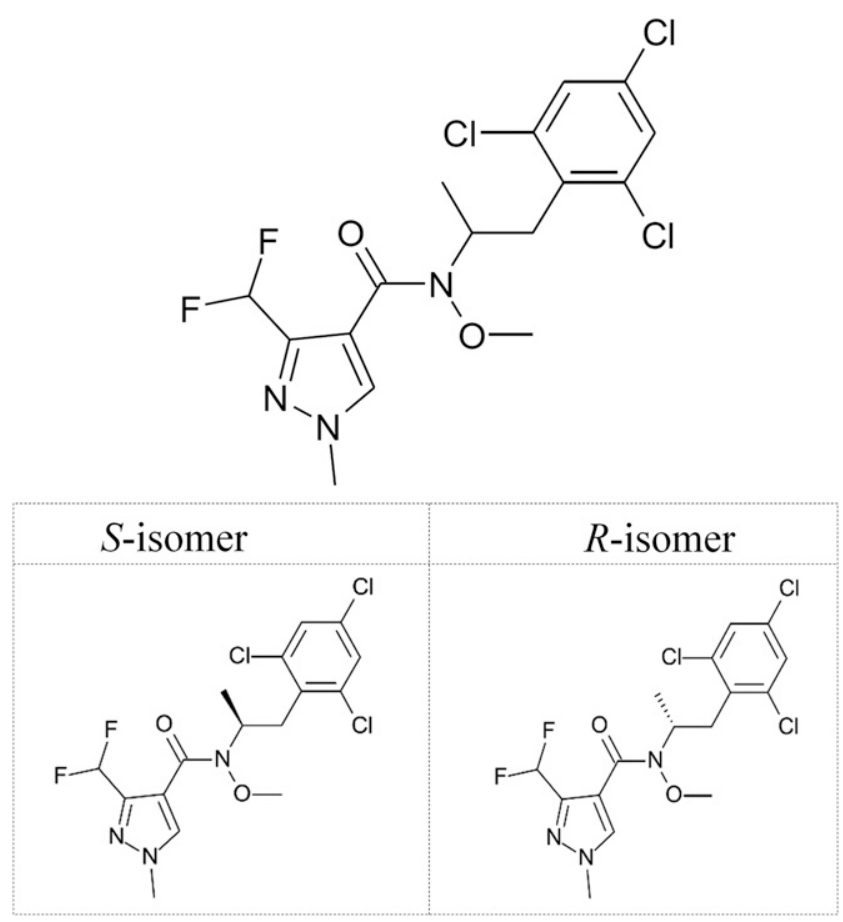

Fig. 1. Chemical structure of pydiflumetofen. Pydiflumetofen consists of two enantiomers: $S$-isomer and $R$-isomer, present in a 1:1 ratio. different concentrations $\left(\mathrm{EC}_{50}\right.$ and $10 \times \mathrm{EC}_{50}$ for each strain) was added into the culture. After culturing for an additional $36 \mathrm{~h}$, the mycelia were harvested and dried and the liquid culture was centrifuged to determine EPS content. EPS content was expressed as a ratio of EPS to mycelial dry weight $(\mathrm{mg} / \mathrm{g})$. Each treatment had four replicates for each strain, and the experiment was repeated twice.

Oxalic acid production. Oxalic acid content as affected by pydiflumetofen was determined as previously described (Duan et al. $2013 \mathrm{~b}$ ). Five mycelial plugs for each strain were transferred to 250-ml conical flasks containing $100 \mathrm{ml}$ of YEPD media. After shaking at $175 \mathrm{rpm}$ for $36 \mathrm{~h}$ at $25^{\circ} \mathrm{C}$, the flasks were amended with pydiflumetofen at the ultimate concentrations of $\mathrm{EC}_{50}$ or $10 \times \mathrm{EC}_{50}$. After shaking for an additional $36 \mathrm{~h}$, the mycelia were harvested and dried, the liquid culture was centrifuged, and the supernatants were used to measure oxalic acid content as previously described (Duan et al. 2013a). The no-fungicide treatments were used as controls. Oxalic acid content was expressed as a ratio of oxalic acid to mycelial dry weight $(\mathrm{g} / \mathrm{g})$. The experiment was repeated twice with four replicates for each treatment.

Efficacy of pydiflumetofen on oilseed rape leaves. Protective and curative effects of pydiflumetofen on detached leaves of oilseed rape against $S$. sclerotiorum were performed according to Duan et al. (2013a). Fresh detached leaves were processed according to the previous method (Wang et al. 2014a, 2016). The leaves of oilseed rape cv. Qinyou 10 were inoculated with the strain HA61. Pydiflumetofen stock solution was diluted to $2.5,5,10,25$, or $50 \mu \mathrm{g} / \mathrm{ml}$ with $0.1 \%$ tween 20. For the protective activity test, pydiflumetofen was sprayed on leaves $24 \mathrm{~h}$ before inoculation with strain HA61. For the curative activity test, pydiflumetofen was sprayed on leaves $24 \mathrm{~h}$ after inoculation with strain HA61. After inoculated leaves were incubated for 4 days at $25^{\circ} \mathrm{C}$ with $80 \%$ relative humidity and a $16 \mathrm{~h}$ photoperiod, the diameters of lesions were measured and control efficacy was calculated according the previous method (Wang et al. 2016). The experiment was repeated twice with 10 replicate leaves for each treatment.

Control efficacy of SSR by pydiflumetofen in the field. To evaluate the effect of pydiflumetofen on controlling SSR, the naturally infected oilseed rape (cv. Ningza 1818) field in Jiangyan City, Jiangsu Province, was used for field trials in 2015 and 2017. Eight treatments including a no-fungicide control were: (1) pydiflumetofen (200 g/liter, SC), $60 \mathrm{~g}$ a.i./ha; (2) pydiflumetofen (200 g/liter, SC), $120 \mathrm{~g}$ a.i./ha; (3) pydiflumetofen (200 g/liter, SC), $180 \mathrm{~g}$ a.i./ha; (4) pydiflumetofen (200 g/liter, SC), $240 \mathrm{~g}$ a.i./ha; (5) carbendazim (50\%, WP), 1,200 g a.i./ha; (6) dimethachlon (50\%, WP), $750 \mathrm{~g}$ a. i./ha; (7) iprodione (40\%, WP), $600 \mathrm{~g}$ a.i./ha; and (8) water control, 750 liters/ha. Each treatment had three replicate plots $\left(20 \mathrm{~m}^{2}\right.$ per plot $)$ that were randomly arranged in the field. The oilseed rape plants were first sprayed with fungicides when $10 \%$ of the major stems were flowering and were sprayed a second time 5 to 7 days later. After about 20 days, disease symptoms of 200 randomly selected plants were visually assessed in each plot and control efficacy was

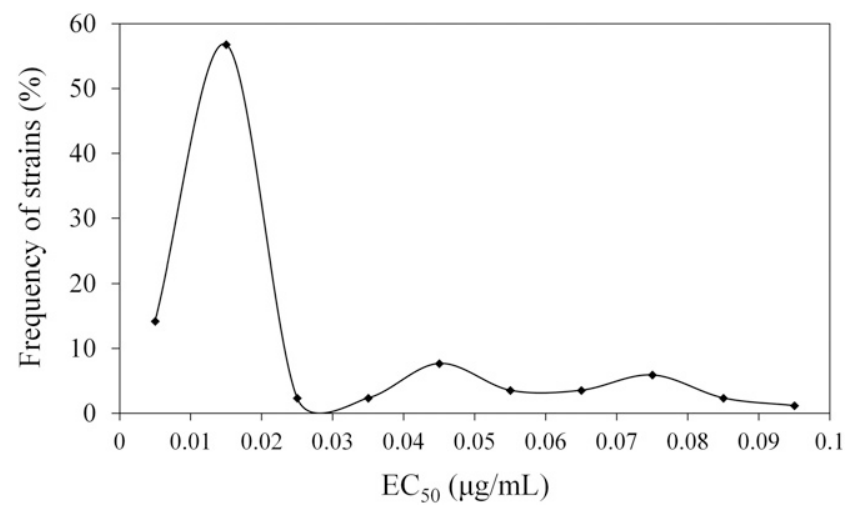

Fig. 2. Sensitivity baseline of 166 Sclerotinia sclerotiorum strains to pydiflumetofen based on in vitro mycelial growth inhibition. 
calculated as previously described (Duan et al. 2012; Wang et al. 2009, 2014a).

Data analysis. Data on relative conductivity, EPS and oxalic acid production of isolates, protective and curative activity, control efficacy of fungicides, and disease index were subjected to analysis of variance. Duncan's new multiple range test was used to assess the differences among the different fungicide treatments or isolates. The Shapiro-Wilk test was used to assess normality of fungicide sensitivity from field isolates. All statistical analyses were performed using IBM SPSS Statistics.

\section{Results}

Pydiflumetofen sensitivity of $S$. Sclerotiorum in vitro. S. Sclerotiorum strains $(n=166)$ were tested for sensitivity to pydiflumetofen based on mycelial growth inhibition. The $\mathrm{EC}_{50}$ values ranged from 0.0058 to $0.0953 \mu \mathrm{g} \mathrm{ml}^{-1}$, and the mean value was $0.025 \mu \mathrm{g} \mathrm{ml}^{-1}$. The sensitivity distribution was unimodal over a sensitive range (Fig. 2). However, the Shapiro-Wilk test indicated that the $\mathrm{EC}_{50}$ values were much wider due to a high variation factor of 16.43 and were not normally distributed $(P<0.05)$. This indicated that pydiflumetofen had a strong inhibitory activity on mycelial growth of $S$. sclerotiorum.

Cross resistance. The $\mathrm{EC}_{50}$ values to pydiflumetofen did not differ among four carbendazim-resistant, four dimethachlon-resistant, and four sensitive strains (Table 1), indicating that no cross resistance was observed between pydiflumetofen and dimethachlon or carbendazim. Therefore, pydiflumetofen should be a good alternative to dimethachlon and carbendazim for controlling S. sclerotiorum.

Cell membrane permeability as affected by pydiflumetofen. With pydiflumetofen treatments or not, the relative conductivity of mycelia for the tested three wild type strains increased over time (Fig. 3). Compared with no-fungicide treated strains, cell membrane permeability of the strains treated with the corresponding $\mathrm{EC}_{50}$ values measured for pydiflumetofen (Table 1) was not significant different (Fig. 3). When the strains were treated with $10 \times \mathrm{EC}_{50}$ values for pydiflumetofen (Table 1), cell membrane permeability significantly increased in comparison with no-fungicide treated controls (Fig. 3 ). The results showed that pydiflumetofen could damage cell membrane permeability in $S$. sclerotiorum, causing an increase in mycelial electrolyte leakage.

EPS production as affected by pydiflumetofen. EPS production of three strains, YZ4024, NT4070, and JY4037, was severely affected with $\mathrm{EC}_{50}$ values of $0.0174,0.0202$, and $0.0323 \mu \mathrm{g} \mathrm{ml}^{-1}$, respectively, measured for pydiflumetofen (Fig. 4). EPS production of those three strains affected with $10 \times \mathrm{EC}_{50}$ values for pydiflumetofen were much lower in comparison with treatment with $\mathrm{EC}_{50}$ values (Fig. 4). This suggested that pydiflumetofen can inhibit EPS production in S. sclerotiorum.

Oxalic acid production as affected by pydiflumetofen. With the presence of pydiflumetofen, oxalic acid content of the three tested $S$. sclerotiorum strains was significantly inhibited in comparison with the corresponding controls (Fig. 5). The inhibition of oxalic acid production increased as the dose of pydiflumetofen increased (Fig. 5). The results indicated that pydiflumetofen could inhibit oxalic acid production, which might be useful for controlling S. sclerotiorum by reducing oxalic acid, a virulent factor in $S$. sclerotiorum.

Protective and curative effects of pydiflumetofen on oilseed rape against $S$. sclerotiorum. Protective and curative activity of pydiflumetofen was measured to assess the potential of pydiflumetofen in controlling SSR on oilseed rape. Pydiflumetofen exerted both protective and curative activity on the detached leaves of oilseed rape inoculated with $S$. sclerotiorum. Protective and curative activity increased as dose increased (Table 2). Control efficacy for protective activity was always higher than that for curative effect at the same

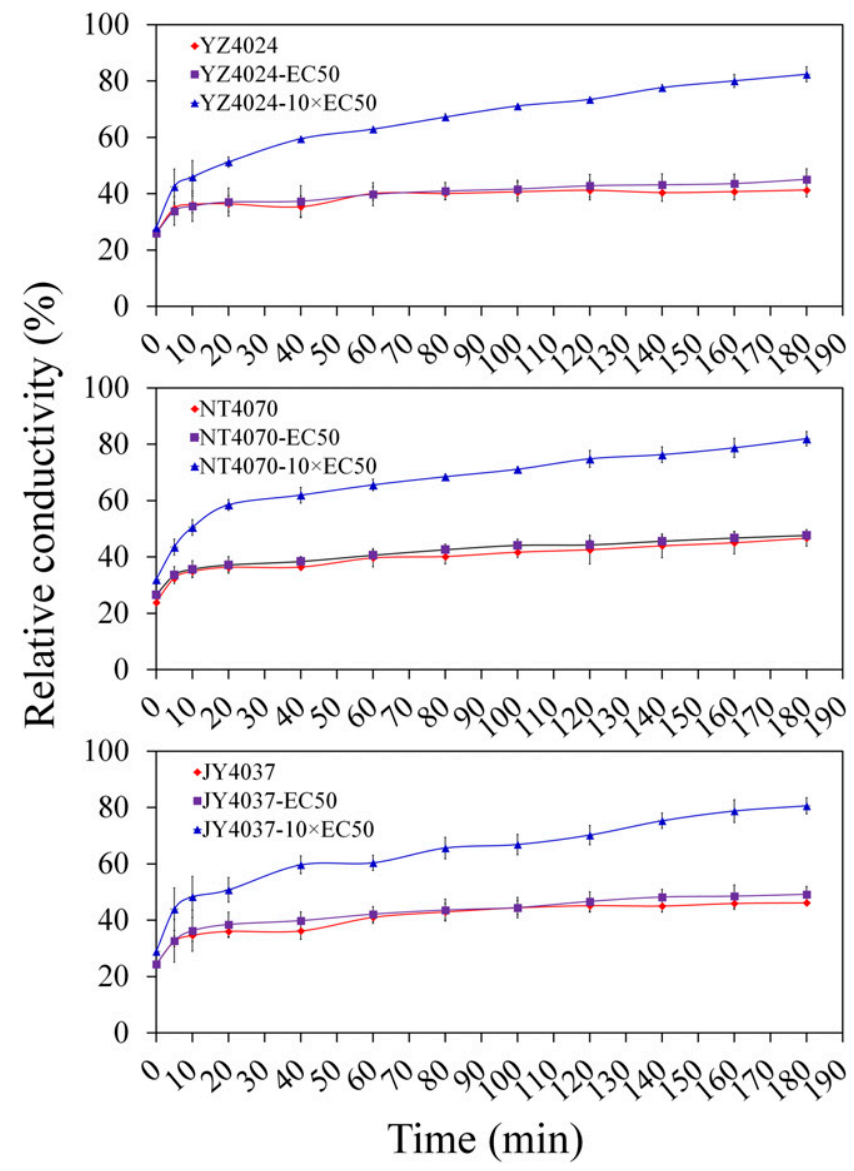

Fig. 3. Relative conductivity of mycelia of three wild type Sclerotinia sclerotiorum strains (YZ4024, NT4070, JY4037) with or without pydiflumetofen treatments. Each panel presents data for each strain.

Table 1. Analysis of cross-resistance between pydiflumetofen and dimethachlon or carbendazim in Sclerotinia sclerotiorum

\begin{tabular}{|c|c|c|c|c|c|}
\hline \multirow[b]{2}{*}{ Strain } & \multirow[b]{2}{*}{ Origin } & \multicolumn{3}{|c|}{$\mathrm{EC}_{50}\left(\mu \mathrm{g} \mathrm{ml}^{-1}\right)$} & \multirow[b]{2}{*}{ Fungicide phenotypes? } \\
\hline & & Carbendazim & Dimethachlon & Pydiflumetofen & \\
\hline YZ4024 & Field & 0.1871 & 0.1863 & 0.0174 & $\mathrm{Car}^{\mathrm{S}} \mathrm{Ipr}^{\mathrm{S}} \mathrm{Pyd}^{\mathrm{S}}$ \\
\hline NT4070 & Field & 0.1374 & 0.1720 & 0.0202 & $\mathrm{Car}^{\mathrm{S}} \mathrm{Ipr}^{\mathrm{S}} \mathrm{Pyd}^{\mathrm{S}}$ \\
\hline JY4037 & Field & 0.2040 & 0.2854 & 0.0323 & $\mathrm{Car}^{\mathrm{S}} \mathrm{Ipr}^{\mathrm{S}} \mathrm{Pyd}^{\mathrm{S}}$ \\
\hline ZJG4035 & Field & 0.1542 & 0.1753 & 0.0160 & $\mathrm{Car}^{\mathrm{S}} \mathrm{Ipr}^{\mathrm{S}} \mathrm{Pyd}^{\mathrm{S}}$ \\
\hline XY4008 & Field & $>100$ & 0.2281 & 0.0238 & $\mathrm{Car}^{\mathrm{R}} \mathrm{Ipr}^{\mathrm{S}} \mathrm{Pyd}^{\mathrm{S}}$ \\
\hline RD4016 & Field & $>100$ & 0.2349 & 0.0183 & $\mathrm{Car}^{\mathrm{R}} \mathrm{Ipr}^{\mathrm{S}} \mathrm{Pyd}^{\mathrm{S}}$ \\
\hline JY4025 & Field & $>100$ & 0.1653 & 0.0231 & $\operatorname{Car}^{\mathrm{R}} \operatorname{Ipr}^{\mathrm{S}} \mathrm{Pyd}^{\mathrm{S}}$ \\
\hline NT4087 & Field & $>100$ & 0.1822 & 0.0227 & $\mathrm{Car}^{\mathrm{R}} \mathrm{Ipr}^{\mathrm{S}} \mathrm{Pyd}^{\mathrm{S}}$ \\
\hline HA61R & Induced in lab & 0.1376 & $>100$ & 0.0103 & $\operatorname{Car}^{\mathrm{S}} \mathrm{Ipr}^{\mathrm{R}} \mathrm{Pyd}^{\mathrm{S}}$ \\
\hline NT18R & Induced in lab & 0.1620 & $>100$ & 0.0177 & $\mathrm{Car}^{\mathrm{S}} \mathrm{Ipr}^{\mathrm{R}} \mathrm{Pyd}^{\mathrm{S}}$ \\
\hline SZ45R & Induced in lab & 0.1238 & $>100$ & 0.0272 & $\mathrm{Car}^{\mathrm{S}} \mathrm{Ipr}^{\mathrm{R}} \mathrm{Pyd}^{\mathrm{S}}$ \\
\hline LYG82R & Field & 0.1483 & $>100$ & 0.0175 & $\mathrm{Car}^{\mathrm{S}} \mathrm{Ipr}^{\mathrm{R}} \mathrm{Pyd}^{\mathrm{S}}$ \\
\hline
\end{tabular}

${ }^{\mathrm{z}} \mathrm{Car}$, Ipr, and Pyd represent carbendazim, iprodione, and pydiflumetofen, respectively; $\mathrm{R}$ and $\mathrm{S}$ represent resistance and sensitivity to fungicide, respectively. 


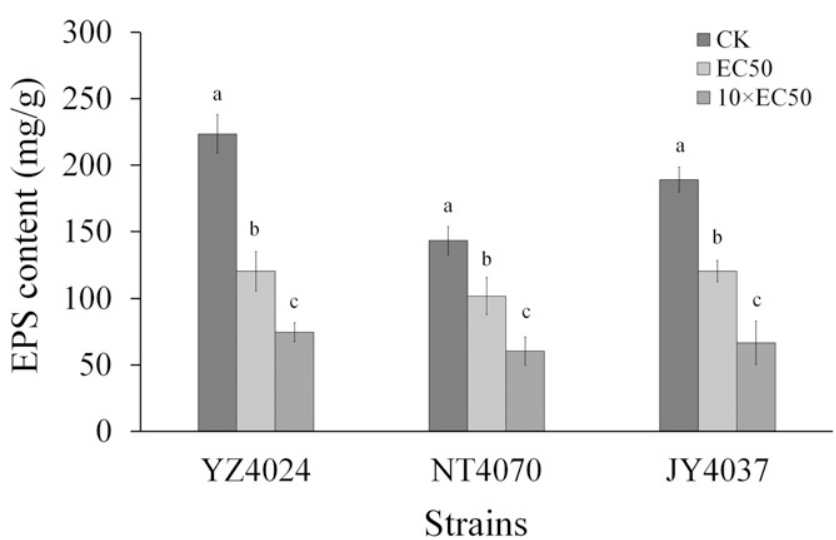

Fig. 4. EPS production of three wild type Sclerotinia sclerotiorum strains (YZ4024, NT4070, JY4037) as affected by pydiflumetofen. EPS content $(\mathrm{mg} / \mathrm{g})$ was represented by a ratio of total EPS to mycelial dry weight. Data represent means \pm SD of three experiments. Bars labeled with different letters with each strain are significantly different with Duncan's new multiple range test at $P=0.05$.

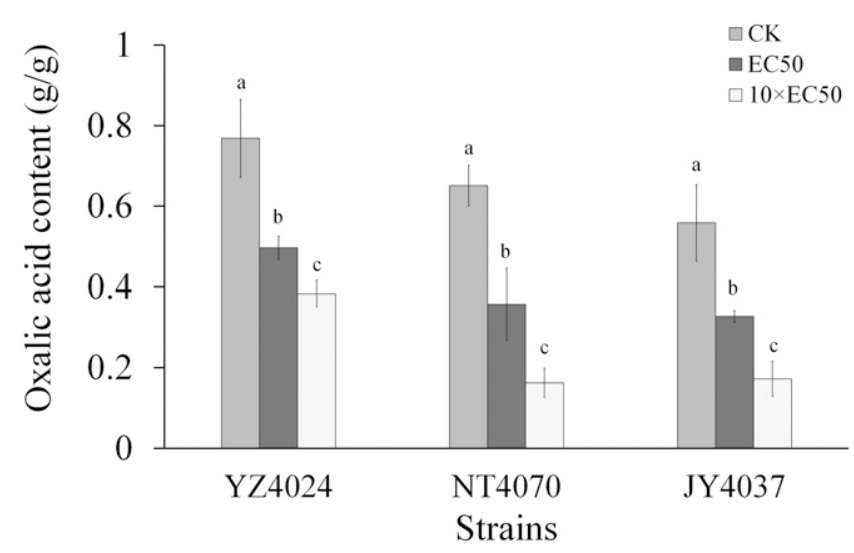

Fig. 5. Oxalic acid production of three Sclerotinia sclerotiorum strains (YZ4024, NT4070, JY4037) as affected by pydiflumetofen. Oxalic acid content (g/g) was represented by a ratio of total oxalic acid to mycelial dry weight. Data represent means \pm SD of three experiments. Bars labeled with different letters with each strain are significantly different with Duncan's new multiple range test at $P=0.05$. concentration of pydiflumetofen (Table 2). This indicated that the protective effect of pydiflumetofen was better than the curative effect in controlling SSR on oilseed rape caused by S. sclerotiorum.

Control efficacy of pydiflumetofen in SSR on oilseed rape in the field. In the field trials, the commonly used fungicides carbendazim, dimethachlon, and iprodione were used as controls. Control efficacy of pydiflumetofen in SSR increased with the increase of the dose (Table 3). Pydiflumetofen at 180 and $240 \mathrm{~g}$ a.i./ha provides substantial control efficacy (Table 3). Dimethachlon and iprodione also controlled SSR, but not as well as pydiflumetofen (Table 3). Carbendazim had the lowest control efficacy on SSR among the fungicide treatments (Table 3). In addition to being highly effective in controlling SSR, pydiflumetofen significantly decreased the fungicide dosage in comparison with commonly used fungicides carbendazim, dimethachlon, and iprodione (Table 3). Therefore, pydiflumetofen not only exhibited a better effect in controlling SSR, but also decreased the dosage of other commonly used fungicides in the field.

\section{Discussion}

In the past four decades, benzimidazoles, mainly carbendazim, have been extensively applied to control SSR on oilseed rape in China. To date, the development of carbendazim-resistant $S$. sclerotiorum populations in the field resulted in control failure of SSR (Duan et al. 2015). Subsequently, dicarboximide fungicides, such as dimethachlon and iprodione, were registered in China to control this disease (Ma et al. 2009b). Unfortunately, dicarboximideresistant S. sclerotiorum populations were discovered in the field only a few years after dicarboximides were used to control this disease (Ma et al. 2009a). Fungicide resistance not only causes a decrease in control efficacy of fungicide on plant diseases, but also increases the dosage of fungicides in the field, worsening environmental pollution. Therefore, searching for new chemical alternatives with different modes of action and high fungicidal activity is necessary for resistance management of $S$. sclerotiorum.

Pydiflumetofen is a novel succinate dehydrogenase inhibitor discovered by Syngenta (Buxton et al. 2016). To date, in vitro fungicidal activity of pydiflumetofen against $S$. sclerotiorum has not been reported. Therefore, in the current research, pydiflumetofen sensitivity of $166 \mathrm{~S}$. sclerotiorum strains was determined based on mycelial growth inhibition. The $\mathrm{EC}_{50}$ values of these strains ranged from 0.0058 to $0.0953 \mu \mathrm{g} \mathrm{ml}^{-1}$ with a mean $\mathrm{EC}_{50}$ value of $0.025 \mu \mathrm{g} \mathrm{ml}^{-1}$. The sensitivity distribution was unimodal, but not normally distributed

Table 2. Protective and curative effects of pydiflumetofen on detached leaves of oilseed rape against Sclerotinia sclerotiorum

\begin{tabular}{|c|c|c|c|c|}
\hline \multirow[b]{2}{*}{ Pydiflumetofen $(\mu \mathrm{g} / \mathrm{ml})$} & \multicolumn{2}{|c|}{ Protective activity } & \multicolumn{2}{|c|}{ Curative activity } \\
\hline & Lesion area $\left(\mathrm{cm}^{2}\right)^{\mathrm{z}}$ & Control efficacy $(\%)^{z}$ & Lesion area $\left(\mathrm{cm}^{2}\right)^{\mathrm{z}}$ & Control efficacy $(\%)^{z}$ \\
\hline 0 & $6.46 \pm 1.44 \mathrm{Aa}$ & I & $7.29 \pm 1.20 \mathrm{Aa}$ & 1 \\
\hline 2.5 & $4.06 \pm 0.48 \mathrm{Bb}$ & $37.23 \pm 7.49 \mathrm{Dd}$ & $5.87 \pm 0.40 \mathrm{Ab}$ & $19.45 \pm 5.44 \mathrm{Dd}$ \\
\hline 5 & $2.77 \pm 0.24 \mathrm{BCc}$ & $57.15 \pm 3.67 \mathrm{Cc}$ & $4.03 \pm 0.67 \mathrm{Bc}$ & $44.72 \pm 9.19 \mathrm{Cc}$ \\
\hline 10 & $1.45 \pm 0.24 \mathrm{CDd}$ & $77.63 \pm 3.77 \mathrm{Bb}$ & $2.11 \pm 0.33 \mathrm{Cd}$ & $71.03 \pm 4.55 \mathrm{Bb}$ \\
\hline 25 & $0.33 \pm 0.15 \mathrm{Dde}$ & $94.90 \pm 2.30 \mathrm{Aa}$ & $1.30 \pm 0.29 \mathrm{Cde}$ & $82.20 \pm 3.99 \mathrm{ABa}$ \\
\hline 50 & $0.21 \pm 0.17 \mathrm{De}$ & $96.69 \pm 2.61 \mathrm{Aa}$ & $0.87 \pm 0.12 \mathrm{Ce}$ & $88.09 \pm 1.58 \mathrm{Aa}$ \\
\hline
\end{tabular}

z Values are the means \pm SD of three experiments. Means followed by different letters within the column are significantly different with Duncan's new multiple range test at $P=0.01$ and $P=0.05$.

Table 3. Control efficacy of SSR as affected by pydiflumetofen in the field in 2015 and 2017

\begin{tabular}{|c|c|c|c|c|c|}
\hline \multirow[b]{2}{*}{ Fungicide } & \multirow[b]{2}{*}{ Dosage (g a.i./ha) } & \multicolumn{2}{|c|}{2015} & \multicolumn{2}{|c|}{2017} \\
\hline & & Disease index ${ }^{z}$ & Control efficacy $(\%)^{\mathrm{z}}$ & Disease index ${ }^{z}$ & Control efficacy $(\%)^{z}$ \\
\hline $200 \mathrm{~g} /$ liter pydiflumetofen (SC) & 60 & $7.69 \pm 0.59 \mathrm{Bc}$ & $59.37 \pm 3.10 \mathrm{Cc}$ & $4.73 \pm 1.04 \mathrm{Cc}$ & $70.90 \pm 6.40 \mathrm{Cb}$ \\
\hline $200 \mathrm{~g} /$ liter pydiflumetofen (SC) & 120 & $3.71 \pm 0.33 \mathrm{Cde}$ & $80.39 \pm 1.75 \mathrm{ABa}$ & $2.77 \pm 0.43 \mathrm{CD} \mathrm{de}$ & $82.99 \pm 2.65 \mathrm{ABa}$ \\
\hline $200 \mathrm{~g} /$ liter pydiflumetofen (SC) & 180 & $2.81 \pm 0.27 \mathrm{Ce}$ & $85.15 \pm 1.44 \mathrm{Aa}$ & $2.30 \pm 0.36 \mathrm{De}$ & $85.88 \pm 2.22 \mathrm{Aa}$ \\
\hline $200 \mathrm{~g} /$ liter pydiflumetofen (SC) & 240 & $2.90 \pm 0.26 \mathrm{Ce}$ & $84.67 \pm 1.35 \mathrm{Aa}$ & $2.13 \pm 0.38 \mathrm{De}$ & $86.93 \pm 2.31 \mathrm{Aa}$ \\
\hline $50 \%$ carbendazim (WP) & 1200 & $9.29 \pm 1.09 \mathrm{Bb}$ & $50.87 \pm 5.75 \mathrm{Dd}$ & $9.26 \pm 0.87 \mathrm{Bb}$ & $43.08 \pm 5.34 \mathrm{Dc}$ \\
\hline $50 \%$ dimethachlon (WP) & 750 & $4.80 \pm 0.45 \mathrm{Cd}$ & $74.64 \pm 2.40 \mathrm{Bb}$ & $4.70 \pm 0.71 \mathrm{Cc}$ & $71.13 \pm 4.37 \mathrm{Cb}$ \\
\hline $40 \%$ iprodione (WP) & 600 & $4.84 \pm 0.32 \mathrm{Cd}$ & $74.40 \pm 1.70 \mathrm{Bb}$ & $4.03 \pm 0.28 \mathrm{CDcd}$ & $75.25 \pm 1.69 \mathrm{BCb}$ \\
\hline Water (CK) & l & $18.92 \pm 1.70 \mathrm{Aa}$ & 1 & $16.27 \pm 1.78 \mathrm{Aa}$ & 1 \\
\hline
\end{tabular}

\footnotetext{
${ }^{\mathrm{z}}$ Values are the means \pm SD of three plots. Means followed by different letters within the column are significantly different with Duncan's new multiple range test
} at $P=0.01$ and $P=0.05$. 
due to a wider variation range of $\mathrm{EC}_{50}$ values. Therefore, sensitivity data are essential for further application of pydiflumetofen in the control of SSR on oilseed rape in the field. Meanwhile, these data also provide important references for subsequent resistance risk assessment and resistance monitoring of $S$. sclerotiorum populations against pydiflumetofen in the future.

As described above, the currently used fungicides, including carbendazim, dimethachlon, and iprodione, which are used in China to control SSR on oilseed rape, have been reported to lead to resistance in S. sclerotiorum (Duan et al. 2016; Wang et al. 2014b). Our previous study reported that the phenylpyrrole fungicide fludioxonil exhibits a highly fungicidal activity in S. sclerotiorum. Surprisingly, fludioxonil has a positive cross resistance to commonly used dicarboximides, such as dimethachlon and iprodione (Duan et al. 2013a; Kuang et al. 2011). To assess whether pydiflumetofen can be used for resistance management in the field, crossresistance between pydiflumetofen and other commonly used fungicides was carried out in this research. As expected, there was no cross resistance between pydiflumetofen and dimethachlon or carbendazim. These results indicated that pydiflumetofen has the potential to be a good alternative to benzimidazoles and dicarboximides for managing fungicide resistance of SSR in the field.

The succinate dehydrogenase (SDH) enzyme, also known as succinate ubiquinone oxidoreductase or complex II, is an essential part of the tricarboxylic cycle and the mitochondrial electron transfer chain; it is the target of SDHIs (Piqueras et al. 2014; Rehfus et al. 2016). The changes in cell membrane permeability in plant pathogenic fungi treated with fungicides was related to increased intracellular plasma leakage. Our findings showed that relative electrical conductivity in mycelia of $S$. sclerotiorum was not affected by a low dose of pydiflumetofen $\left(\mathrm{EC}_{50}\right)$. After treatment with a high dose of pydiflumetofen $\left(10 \times \mathrm{EC}_{50}\right)$, however, relative electrical conductivity significantly increased compared with the controls, indicating an increase in cell membrane permeability. At the low dose, pydiflumetofen can partly inhibit the mitochondrial electron transfer chain and the tricarboxylic cycle, but does not disrupt cell membrane permeability. With the increase in dose, however, pydiflumetofen increases the inhibitory effect on mycelial growth, which can disorder cell metabolism, causing the change of cell membrane permeability in $S$. sclerotiorum. This showed that pydiflumetofen can indirectly impair the membrane structure of $S$. sclerotiorum by affecting the mitochondrial electron transfer chain and the tricarboxylic cycle. Exopolysaccharide production has been used as an indicator of virulence in Xanthomonas species and other pathogens (Barrére et al. 1986; Dharmapuri et al. 2001; Leigh and Coplin 1992). Oxalic acid has been demonstrated to play a vital role in infection progress and fungal development in S. sclerotiorum (Dutton and Evans 1996; Godoy et al. 1990). In this research, EPS and oxalic acid content significantly decreased after pydiflumetofen treatment, which might contribute to the decreased virulence of pathogens and result in failure of infection. Our findings are largely consistent with the effect of fludioxonil in S. sclerotiorum (Duan et al. 2013a). However, it is not clear how pydiflumetofen inhibits biosynthesis of EPS and oxalic acid in S. sclerotiorum; therefore, further analysis of the phenomenon will shed light on the understanding of not only molecular action mode but also resistance mechanism of S. sclerotiorum to pydiflumetofen.

To explore the application of pydiflumetofen in controlling SSR on oilseed rape, artificially inoculated experiments and naturally infected field trials were performed in this study, respectively. On detached oilseed rape leaves, pydiflumetofen exhibited excellent protective and curative effects, and protective effect was better than curative effect. Similar results were also observed with other fungicides (Duan et al. 2013a; Wang et al. 2014a, 2016). These findings provide a novel insight into understanding the timing of fungicide application for controlling plant diseases. In the field trials, control efficacy of pydiflumetofen in SSR on oilseed rape was better than that of the commonly used fungicides carbendazim, dimethachlon, and iprodione. Among the fungicide treatments, carbendazim recorded minimum control efficacy in SSR on oilseed rape. Thus, we suspect that low control efficacy by carbendazim might result from carbendazim resistance in our experimental fields. Therefore, we further monitored the frequency of benzimidazole- and dicarboximideresistant $S$. sclerotiorum populations from the experimental fields in 2015 and 2017, respectively. No dicarboximide resistance was detected, but benzimidazole resistance was very severe, and benzimidazoleresistant frequency of S. sclerotiorum populations reached 45.32 and $53.18 \%$ in 2015 and 2017, respectively (data not shown), causing the decrease of control efficacy of carbendazim on SSR in our field trials. These results further demonstrated that benzimidazole resistance led to the decrease of control efficacy in the field. Considering its excellent in vitro fungicidal effect on S. sclerotiorum, better control efficacy on SSR in the field, and no cross resistance with current commonly used fungicides, pydiflumetofen has a potential application for management of SSR on oilseed rape caused by benzimidazole- and dicarboximide-resistant populations.

\section{Literature Cited}

Abawi, G. S., and Grogan, R. G. 1979. Epidemiology of diseases caused by Sclerotinia species. Phytopathology 69:899-904.

Bardin, S. D., and Huang, H. C. 2001. Research on biology and control of Sclerotinia diseases in Canada. Can. J. Plant Pathol. 23:88-98.

Barrére, G. C., Barber, C. E., and Daniels, M. J. 1986. Molecular cloning of genes involved in the production of the extracellular polysaccharide xanthan by Xanthomonas campestris pv. campestris. Int. J. Biol. Macromol. 8:372-374.

Boland, G. J. 1997. Stability analysis for evaluating the influence of environment on chemical and biological control of white mold (Sclerotinia sclerotiorum) of bean. Biol. Control 9:7-14.

Boland, G. J., and Hall, R. 1994. Index of plant hosts of Sclerotinia sclerotiorum. Can. J. Plant Pathol. 16:93-108.

Bolton, M. D., Thomma, B. P. H. J., and Nelson, B. D. 2006. Sclerotinia sclerotiorum (Lib.) de Bary: biology and molecular traits of a cosmopolitan pathogen. Mol. Plant Pathol. 7:1-16.

Buxton, K., Harp, T., Tally, A., and Mclean, H. 2016. Adepidyn (TM): A new fungicide active ingredient for control of foliar diseases. Phytopathology (suppl.) 106:S4.61.

Dharmapuri, S., Yashitola, J., Vishnupriya, M. R., and Sonti, R. V. 2001. Novel genomic locus with atypical $\mathrm{G}+\mathrm{C}$ content that is required for extracellular polysaccharide production and virulence in Xanthomonas oryzae pv. oryzae. Mol. Plant-Microbe Interact. 14:1335-1339.

Duan, Y., Ge, C., Liu, S., Chen, C., and Zhou, M. 2013a. Effect of phenylpyrrole fungicide fludioxonil on morphological and physiological characteristics of Sclerotinia sclerotiorum. Pestic. Biochem. Physiol. 106:61-67.

Duan, Y., Ge, C., Liu, S., Wang, J., and Zhou, M. 2013b. A two-component histidine kinase Shk1 controls stress response, sclerotial formation and fungicide resistance in Sclerotinia sclerotiorum. Mol. Plant Pathol. 14: 708-718.

Duan, Y., Liu, S., Ge, C., Feng, X., Chen, C., and Zhou, M. 2012. In vitro inhibition of Sclerotinia sclerotiorum by mixtures of azoxystrobin, SHAM, and thiram. Pestic. Biochem. Physiol. 103:101-107.

Duan, Y. B., Ge, C. Y., and Zhou, M. G. 2014. Molecular and biochemical characterization of Sclerotinia sclerotiorum laboratory mutants resistant to dicarboximide and phenylpyrrole fungicides. J. Pest Sci. 87:221-230.

Duan, Y. B., Yang, Y., Wang, J. X., Liu, C. C., He, L. L., and Zhou, M. G. 2015. Development and application of loop-mediated isothermal amplification for detecting the highly benzimidazole-resistant isolates in Sclerotinia sclerotiorum. Sci. Rep. 5:17278.

Duan, Y. B., Yang, Y., Wang, Y., Pan, X. Y., Wu, J., Cai, Y. Q., Li, T., Zhao, D. L., Wang, J. X., and Zhou, M. G. 2016. Loop-mediated isothermal amplification for the rapid detection of the F200Y mutant genotype of carbendazimresistant isolates of Sclerotinia sclerotiorum. Plant Dis. 100:976-983.

Dutton, M. V., and Evans, C. S. 1996. Oxalate production by fungi: its role in pathogenicity and ecology in the soil environment. Can. J. Microbiol. 42: 881-895.

Ge, C. Y., Duan, Y. B., Zhou, M. G., and Chen, C. J. 2013. A protoplast transformation system for gene deletion and complementation in Sclerotinia sclerotiorum. J. Phytopathol. 161:800-806.

Godoy, G., Steadman, J. R., Dickman, M. B., and Dam, R. 1990. Use of mutants to demonstrate the role of oxalic acid in pathogenicity of Sclerotinia sclerotiorum on Phaseolus vulgaris. Physiol. Mol. Plant Pathol. 37:179-191.

Harp, T., Anaka, K., and Tally, A. 2016. Adepidyn (TM): A new fungicide active ingredient for control of Fusarium head blight on wheat. Phytopathology (suppl.) 106:S4.61.

Hou, Y. P., Mao, X. W., Wang, J. X., Zhan, S. W., and Zhou, M. G. 2017. Sensitivity of Fusarium asiaticum to a novel succinate dehydrogenase inhibitor fungicide pydiflumetofen. Crop Prot. 96:237-244.

Kuang, J., Hou, Y. P., Wang, J. X., and Zhou, M. G. 2011. Sensitivity of Sclerotinia sclerotiorum to fludioxonil: In vitro determination of baseline sensitivity and resistance risk. Crop Prot. 30:876-882. 
Lee, Y. H., Cho, Y. S., Lee, S. W., and Hong, J. K. 2012. Chemical and biological controls of balloon flower stem rots caused by Rhizoctonia solani and Sclerotinia sclerotiorum. Plant Pathol. J. 28:156-163.

Leigh, J. A., and Coplin, D. L. 1992. Exopolysaccharides in plant-bacterial interactions. Annu. Rev. Microbiol. 46:307-346.

Letham, D. B., Huett, D. O., and Trimboli, D. S. 1976. Biology and control of Sclerotinia sclerotiorum in cauliflower and tomato crops in coastal New South Wales. Plant Dis. Rep. 60:286-289.

Li, H. X., Lu, Y. J., and Zhou, M. G. 2003. Mutation in $\beta$-tubulin of Sclerotinia sclerotiorum conferring resistance to carbendazim in rapeseed field isolates. Chin. J. Oil Crop Sci. 2:56-60.

Ma, H. X., Feng, X. J., Yu, C., Chen, C. J., and Zhou, M. G. 2009a. Occurrence and characterization of dimethachlon insensitivity in Sclerotinia sclerotiorum in Jiangsu Province of China. Plant Dis. 93:36-42.

Ma, H. X., Yu, C., Wang, J. X., Yu, W. Y., Tang, Z. H., Chen, C. J., and Zhou, M. G. 2009b. Activity of carbendazim, dimethachlon, iprodione, procymidone and boscalid against Sclerotinia stem rot in Jiangsu Province of China. Phytoparasitica 37:421-429.

Olaya, G., Linley, R., Edlebeck, K., and Harp, T. 2016. ADEPIDYN (TM) fungicide: Cross resistance patterns in Alternaria solani. Phytopathology (suppl.) 106:S4.12
Piqueras, C. M., Latorre, B. A., and Torres, R. 2014. Effectiveness of isofetamid, a new succinate dehydrogenase inhibitor fungicide, in the control of grapevine gray mold. Cienc. Investig. Agrar. 41:365-374.

Purdy, L. H. 1979. Sclerotinia sclerotiorum: history, diseases and symptomatology, host range, geographic distribution, and impact. Phytopathology 69: 875-880.

Rehfus, A., Miessner, S., Achenbach, J., Strobel, D., Bryson, R., and Stammler, G. 2016. Emergence of succinate dehydrogenase inhibitor resistance of Pyrenophora teres in Europe. Pest Manag. Sci. 72:1977-1988.

Wang, J. X., Ma, H. X., Chen, Y., Zhu, X. F., Yu, W. Y., Tang, Z. H., Chen, C. J., and Zhou, M. G. 2009. Sensitivity of Sclerotinia sclerotiorum from oilseed crops to boscalid in Jiangsu Province of China. Crop Prot. 28:882-886.

Wang, Y., Duan, Y. B., and Zhou, M. G. 2014a. Control of Sclerotinia sclerotiorum infection in oilseed rape with strobilurin fungicide SYP-7017. Can. J. Plant Pathol. 36:354-359.

Wang, Y., Duan, Y. B., and Zhou, M. G. 2016. Baseline sensitivity and efficacy of fluazinam in controlling Sclerotinia stem rot of rapeseed. Eur. J. Plant Pathol. 144:337-343.

Wang, Y., Hou, Y. P., Chen, C. J., and Zhou, M. G. 2014b. Detection of resistance in Sclerotinia sclerotiorum to carbendazim and dimethachlon in Jiangsu Province of China. Australas. Plant Pathol. 43:307-312. 